\title{
The three-dimensional structure of sunspots
}

\section{The moat flow at two different heights}

\author{
H. Balthasar ${ }^{1}$ and K. Muglach ${ }^{2}$ \\ 1 Astrophysikalisches Institut Potsdam, An der Sternwarte 16, 14482 Potsdam, Germany \\ e-mail: hbalthasar@aip.de \\ 2 Artep. Inc., Naval Research Laboratory, 4555 Overlook Ave. SW, Washington DC 20375, USA \\ e-mail: muglach@nrl.navy.mil
}

Received 24 July 2009 / Accepted 2 November 2009

\section{ABSTRACT}

\begin{abstract}
Aims. Many sunspots are surrounded by a radial outflow called the moat flow. We investigate the moat flow at two different heights of the solar atmosphere for a sunspot whose magnetic properties were reported in the first paper of this series.

Methods. We use two simultaneous time series taken with the Transition Region And Coronal Explorer (TRACE) in white light and in the UV at $170 \mathrm{~nm}$. The field-of-view is centered on the small sunspot NOAA 10886 located near disk center. Horizontal velocities are derived by applying two different local correlation tracking techniques.

Results. Outflows are found everywhere in the moat. In the inner moat, the velocities from the UV series are larger than those from white light, whereas in the outer part of the moat we find the converse result.

Conclusions. The results imply that the white light velocities represent a general outflow of the quiet sun plasma in the moat, while UV velocities are dominated by small bright points that move faster than the general plasma flow.
\end{abstract}

Key words. sunspots - Sun: UV radiation

\section{Introduction}

A horizontal outflow around sunspots, called the moat flow, was first detected by Sheeley (1972) while investigating the Doppler shift of a magnetically insensitive spectral line (Fe I $543.4 \mathrm{~nm}$ ). Its existence was confirmed spectroscopically by Balthasar et al. (1996). The speed of these outflows is on the order of $0.5-1.0 \mathrm{~km} \mathrm{~s}^{-1}$.

Near-disk-center horizontal motions cannot be detected spectroscopically, but local correlation tracking (LCT) techniques introduced by November \& Simon (1988) allow us to follow the proper motions of intensity structures such as granules or G-band bright points. Applying LCT, Rimmele (1997) observed a maximum speed of $1 \mathrm{~km} \mathrm{~s}^{-1}$. Sobotka \& Roudier (2007) investigated time series of 32 sunspots being observed in white light (WL) with the Transition Region And Coronal Explorer (TRACE), 26 of these sunspots were observed simultaneously in the UV at $160 \mathrm{~nm}$. They found that moat flows occur in both young and old spots. In older spots, the moat is more extended, and the flow velocity is $0.410 \mathrm{~km} \mathrm{~s}^{-1}$ instead of $0.380 \mathrm{~km} \mathrm{~s}^{-1}$ for young spots. On average, velocities are higher by $0.04-0.07 \mathrm{~km} \mathrm{~s}^{-1}$ in the UV. Vargas Domínguez et al. (2007, 2008) found moat flows on the order of $0.7 \mathrm{~km} \mathrm{~s}^{-1}$, but only where the adjacent penumbra has a radial structure. Locations without penumbra or with a non-radial penumbra have no moat flow. On the other hand, Deng et al. (2007) observed a persisting moat flow after the penumbra of a decaying sunspot had disappeared.

LCT can also be applied to small magnetic areas that are found in the moat moving outward. They are called moving magnetic features (MMFs, see Harvey \& Harvey 1973) and were observed first by Sheeley (1969). Sheeley discovered that these features coincide with bright points in the $\mathrm{CN}$-bandhead close to $388.3 \mathrm{~nm}$. MMFs were studied by Brickhouse \& LaBonte (1988), who measured velocities of about $0.5 \mathrm{~km} \mathrm{~s}^{-1}$. They claimed that the moat radius is roughly twice the penumbral radius. Hagenaar \& Shine (2005) found that MMFs begin at high velocities of $1.8 \mathrm{~km} \mathrm{~s}^{-1}$ near the outer boundary of the penumbra, which is faster than average moat velocities. Zhang et al. (2007) also found an outward decrease in flow velocity of MMFs from $0.6 \mathrm{~km} \mathrm{~s}^{-1}$ to $0.35 \mathrm{~km} \mathrm{~s}^{-1}$. Sainz Dalda \& Martínez Pillet (2005) and Ravindra (2006) found that MMFs originate in the outer penumbra. Intergranular G-band bright points also exhibit an outward motion near the spot, at least in their majority. Bovelet $\&$ Wiehr (2003) reported velocities on the order of $0.3 \mathrm{~km} \mathrm{~s}^{-1}$ close to the penumbral boundary.

Kubo et al. (2007) detected horizontal velocities of up to $1.5 \mathrm{~km} \mathrm{~s}^{-1}$ by investigating the magnetic properties of MMFs. Choudhary \& Balasubramaniam (2007) found that there are fewer MMFs in the lower chromosphere than in the photosphere. Lin et al. (2006) identified an MMF with brightenings in the chromosphere and transition region and concluded that MMFs influence the dynamics in the upper layers of the solar atmosphere. Zuccarello et al. (2009) observed MMFs streaming outward from a sunspot that had not developed a penumbra.

Waves propagating outward in areas outside the spots were detected by Georgakilas et al. (2000) from observations in the Fe I $557.6 \mathrm{~nm}$ line. These waves have a phase velocity of about $0.5 \mathrm{~km} \mathrm{~s}^{-1}$. Balthasar \& Schleicher (2008) discovered Dopplervelocity features that survive for $45 \mathrm{~min}$ or even longer, traveling 
throughout the moat. These features are restricted to the moat, not being detectable outside the moat.

In this paper, we investigate a time series of a small sunspot and its surroundings observed in two wavelength regimes with the spacecraft TRACE. White-light images provide insight into the photosphere, and images at $170 \mathrm{~nm}$ correspond to the layer just below the temperature minimum. The magnetic properties of the same sunspot were determined by Balthasar \& Gömöry (2008, hereafter Paper I). This is an important difference from the investigations of Sobotka \& Roudier (2007) who applied a similar method as ourselves, but had no highly resolved magnetic data of their spots.

\section{Observations and data reduction}

We investigate two simultaneous time series obtained with TRACE on May 27, 2006. For a detailed description of the instrument, we refer to Handy et al. (1999). One series is in $\mathrm{WL}$ and the other in ultraviolet at $170 \mathrm{~nm}$ (UV). The WL filter covers a wide wavelength range and reveals the layer at which the quiet sun's continuum at $500 \mathrm{~nm}$ originates. According to Vernazza et al. (1981), the UV continuum at $170 \mathrm{~nm}$ is formed 450-500 km higher in the quiet sun, just below the temparature minimum. Uitenbroek (2004) computed the optical depth unity for the $170 \mathrm{~nm}$ continuum across a cross-section in a $3 \mathrm{~d}$ snapshot of a simulation of solar convection. The height of optical depth $\tau=1$ varies between about 150 and $350 \mathrm{~km}$ and is, on average, located around $250 \mathrm{~km}$.

The field-of-view was centered on a small sunspot, NOAA 10886, which was passing the central meridian at a heliographic latitude of $7^{\circ} \mathrm{N}$. This spot had a radius of $6.7 \mathrm{Mm}$. The series started at 00:39:30 UT, and had a cadence of $1 \mathrm{~min}$ and a total duration of $6 \mathrm{~h}$ and $41 \mathrm{~min}$. Moats can persist for this length of time, e.g., Sobotka \& Roudier (2007) found moats that are stable over a period of $12 \mathrm{~h}$.

Data reduction largely followed that described in detail in Muglach (2003). We subtracted a background and applied a flatfield to correct for the loss of sensitivity of the central part of the CCD. The image sequences were compensated for differential solar rotation and the orbital image drift was taken care of using cross-correlation methods. To remove intensity fluctuations caused by p-mode oscillations, we also applied a $k-\omega$ filter to the WL series with a cutoff phase velocity of $v_{\mathrm{ph}}=3 \mathrm{~km} \mathrm{~s}^{-1}$.

Figure 1 shows a selected area containing the spot. The size of the area is $400 \times 400$ pixel, and the size of a pixel is 0.5 corresponding to $370 \mathrm{~km}$. Outside the spot, the WL-images are dominated by the granular structure, which serves as a tracer for LCT. The single UV-images show many bright points moving radially with respect to the center of the spot, indicated by the elongated features in the averaged image.

To determine horizontal motions, we employ two different LCT techniques. Here we compare the results of two different LCT codes. The first is a classical LCT code (CLCT) that is the Lockheed Martin's Solar and Astrophysical Laboratory's version based on the original works of November \& Simon (1988). This method was used before e.g., by Deng et al. (2006, 2007). The second method is Fourier local correlation tracking (FLCT) presented by Fisher \& Welsch (2008). An extensive comparison of these two and several other LCT methods based on synthetic magnetograms is presented by Welsch et al. (2007). With their selection of parameters, they achieved a slightly superior performance for FLCT compared to CLCT (see their Fig. 6).

We correlate images with a time difference of eight minutes thus producing 401 velocity maps, which we average to obtain the final velocity map. Both LCT methods need a spatial parameter as input, which preselects the spatial scales on which the horizontal velocities are determined. For CLCT, one has to select the width of the correlation box. We selected the values for $x$ and $y$ identically and tried different values. The value of four pixels corresponding to $1.47 \mathrm{Mm}$ seems to be the most suitable selection for us because this value fits the size of granules in WL and that of the bright features in UV. A Gaussian bell, which has a width of 1.5 times the box size, is applied to the boxes as a weighting function. Shifts greater than $60 \%$ of the box size, but not smaller than three pixels, are clipped. FLCT also uses a Gaussian windowing function. Each pixel is multiplied by a Gaussian of width $\sigma$, where $\sigma$ is chosen by the user. The final box size is determined by a cut-off value of the image, which is set to be 0.1 . In this way, the final box size is a factor of $\sim 3$ greater than the width of the Gaussian. A detailed description of the computational approach including examples and runtime information of the FLCT is given by Fisher \& Welsch (2008). The software is public and can be downloaded ${ }^{1}$.

Both methods give estimates of the horizontal motions in both the $x$ and $y$ direction. These values are transformed into both a radial and tangential component using the equations

$v_{\mathrm{r}}=v_{x} \cos \varphi+v_{y} \sin \varphi$ and

$v_{\mathrm{t}}=-v_{x} \sin \varphi+v_{y} \cos \varphi$

where $\varphi$ is the azimuth angle measured about the center of the spot.

\section{Results}

\subsection{Comparison of white light and UV series}

Horizontal flows were determined independently for the WL and the UV series. Maps of the radial and tangential velocities derived by CLCT are shown in Fig. 2 for a box width of four pixels. We detect radial outflows everywhere in the moat for both wavelength ranges. Velocities are higher in the UV for the inner moat, while WL exhibits higher velocities in the outer moat. For the inner moat, our detection of higher velocities in UV agrees with the results of Sobotka \& Roudier (2007). Most of the structures that we track in WL are granules with a short lifetime. Thus, it is not surprising that we see small-scale structures in the WL-velocities. The bright points in the UV images survive for longer, and we find more elongated structures in the UVvelocities. Tangential flows are rather small in the moat. There is a weak tendency for a negative value in WL, which would imply with a clockwise motion. The radial dependence of the velocities after averaging over the azimuth with respect to the center of the spot is depicted in Fig. 3. The outflow of the moat ends at $27 \mathrm{Mm}$ for both WL and UV images. The moat radius of $27 \mathrm{Mm}$ is roughly four times the spot radius of 6.7 Mm. Here, we observe a quite extended moat compared to the statistical values published by Brickhouse \& LaBonte (1988) and Sobotka \& Roudier (2007). The extent of the flow coincides quite well with the distance to plage areas and network points next to the spot as seen in the UV image. These structures can also be recognized in the magnetic maps in Paper I. Therefore, we consider this distance to represent the outer boundary of the moat.

In the penumbra, we see a few locations with inward motions. Obviously this is not caused by penumbral grains that are known to move outward in the outer penumbra. Their size is

\footnotetext{
1 http://solarmuri.ssl . berkeley.edu/overview/ publicdownloads/software.html
} 
H. Balthasar and K. Muglach: The three-dimensional structure of sunspots. II.

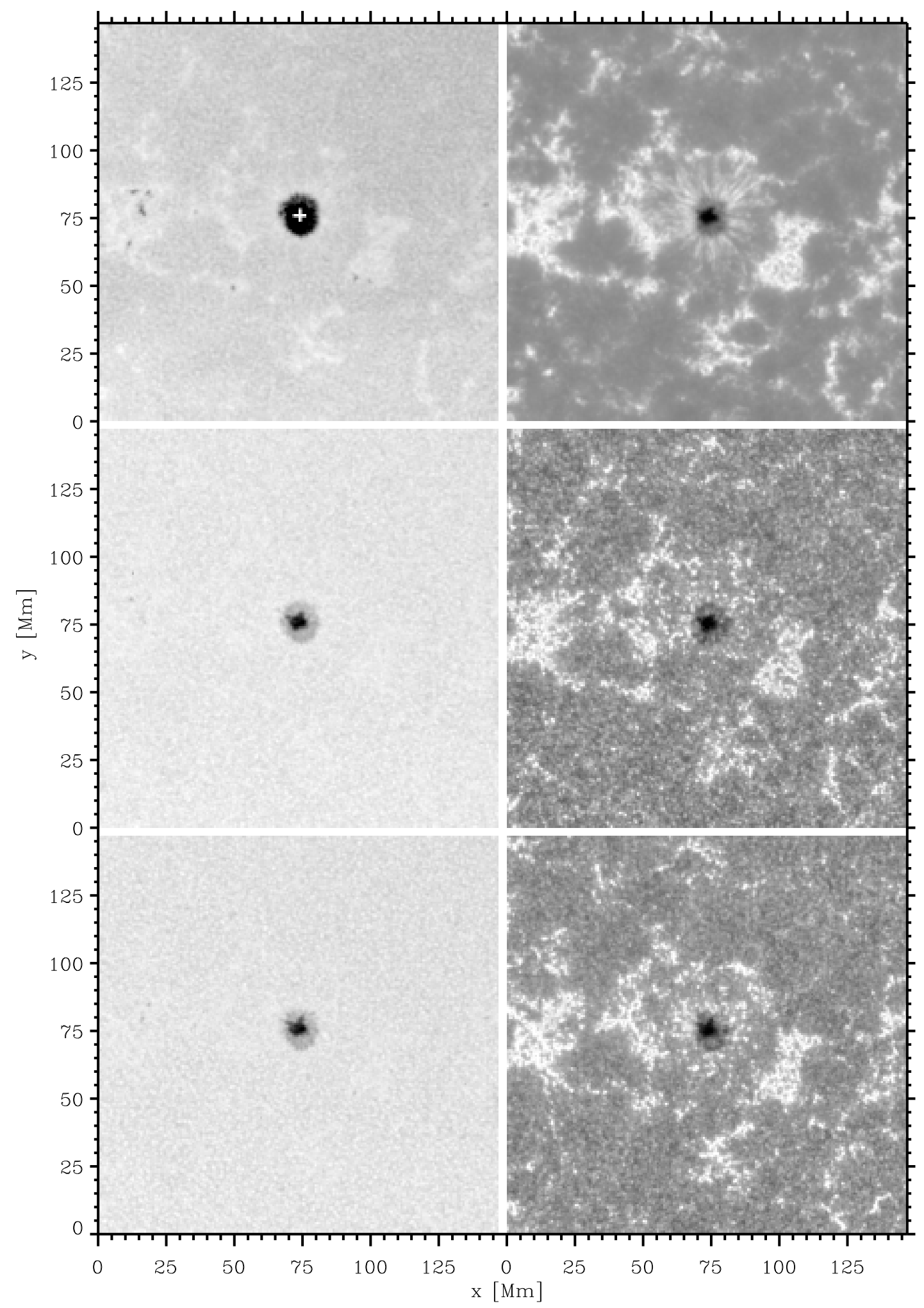

Fig. 1. Images of the observed sunspot and its surroundings. White light images are given in the left column and UV images at $170 \mathrm{~nm}$ in the right one. The upper row shows images averaged over the complete time series, the middle row shows images at the start of the series, and the bottom row those at the end. The dark parts of the spot are suppressed in the averaged WL image to enhance the visibility of bright network structures that delineate the moat. UV images are displayed on a logarithmic scale. The white cross marks the center of the spot. The center of the solar disk is towards the lower edge.

probably too small (below the TRACE resolution) to affect our results.

Beyond $27 \mathrm{Mm}$, the WL curve shows a flow towards the spot, while the UV curve remains close to zero. The moat is surrounded by supergranules that manifest themselves as horizontal outflows from their respective centers. Therefore, it is to be expected that the moat is surrounded by motions toward the spot, as we see in WL. At a distance of roughly $50 \mathrm{Mm}$, 


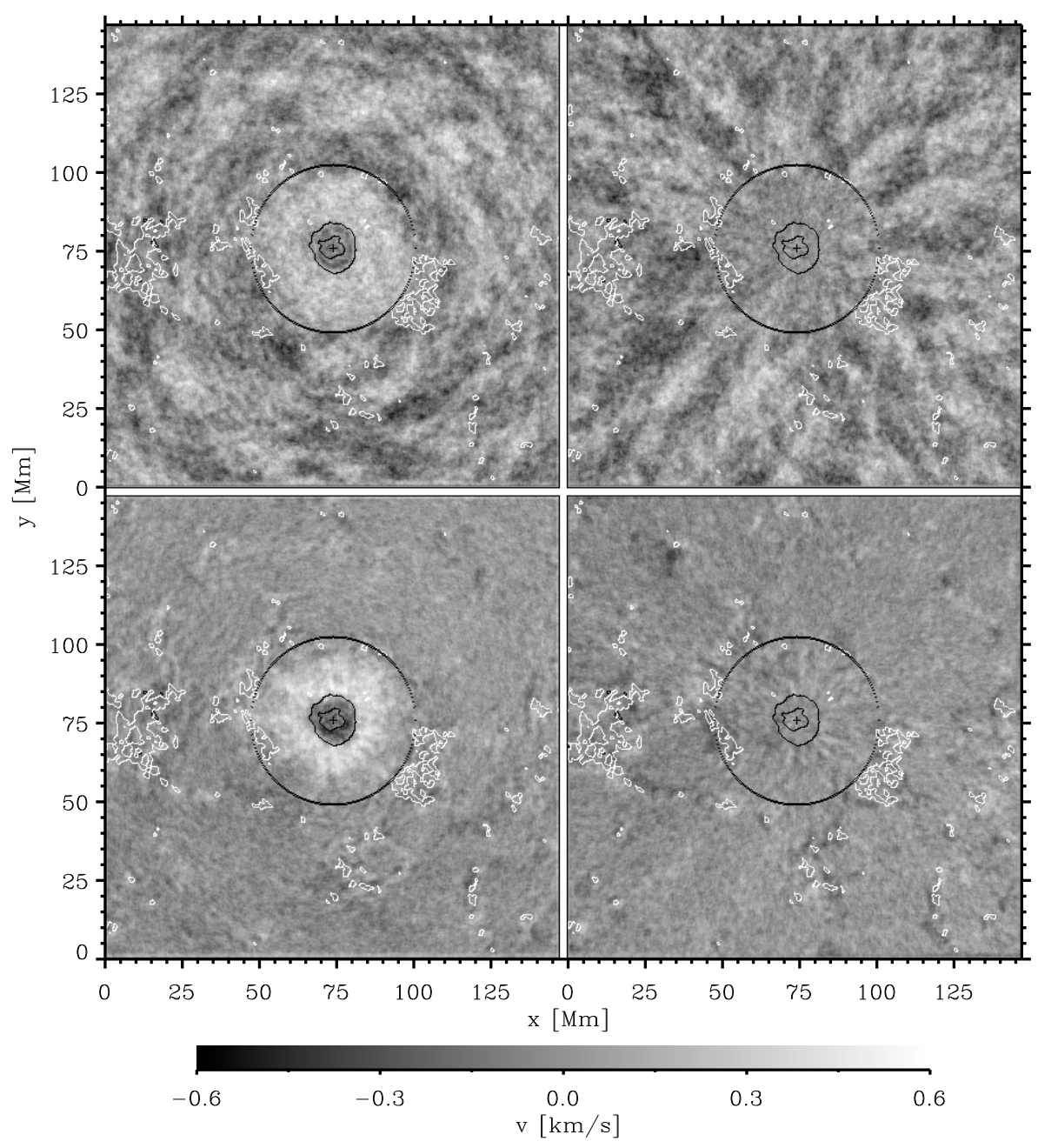

Fig. 2. Radial (left) and tangential (right) horizontal velocities for the WL series (top) and the UV series (bottom), obtained from CLCT with a box size of four pixels. The dark lines mark the outer boundaries of the umbra and penumbra. The extension of the moat is given by the circle with a radius of $27 \mathrm{Mm}$. The white contours indicate the bright structures seen in the UV. The direction to disk center is downwards. Positive velocities are outward and counterclockwise, respectively. on the far side of the supergranules, we detect another ring of outflow. These alternations repeated several times with increasing distance from the spot, but the relation to the spot becomes less pronounced. No systematic flow can be detected in UV close to the moat. The supergranular flow is no longer present in those layers in which the UV light originates. The supergranular flows also dominate the maps of tangential WL velocities outside the moat. Supergranules alternate between positive and negative velocities in the tangential direction, and this pattern is reversed in the next ring of supergranules. For this spot, we do not detect significant asymmetry in the east-west direction as found for other ones by Sobotka \& Roudier (2007).

\subsection{Comparison of the two methods}

To compare the two methods, the parameters must be selected in such a way that differences caused by this selection are minimized. Using CLCT, one chooses the box size as the main parameter, and for FLCT the main parameter is the width $\sigma$ of the Gaussian bell. The corresponding selection for CLCT is a box size of 12 pixels and a factor of 0.33 for the Gaussian bell. In Figs. 4 and 5, we compare the results of the two methods. The large-scale structures in the velocity field are similar for both methods, but CLCT velocities cover a wider range. The spatial resolution is of course lower than for a box size of four pixels as e.g., in Fig. 2, but surprisingly we measure higher velocities for the larger box size. For both methods, we see the outflow that covers the entire moat. Beyond the moat, we detect in WL the ring-like structures of positive and negative radial velocity components and the alternation in the signs of the velocity for the tangential component, which are signatures of the supergranular velocity field.

Outside the moat, results from CLCT applied to the UV data exhibit a patchy structure that resembles that of solar oscillations with typical lengths of about $5^{\prime \prime}$. Superimposed is a faint indication of ring segments in the radial component and a radial structure in the tangential one, but the width of these structures is smaller than in the WL data.

To present the difference more quantitatively, we calculated linear regression fits of the radial velocities from FLCT compared to those from CLCT. Here, we concentrate on the moat and therefore suppress all values both inside the spot and beyond the mean moat radius of $27 \mathrm{Mm}$. Coefficients of these fits are given in Table 1. Scatter diagrams for the box size of 12 pixels are shown in Figs. 6 and 7. Regression coefficients are smaller than one, indicating that CLCT velocities are generally higher than FLCT velocities, but the scatter shows that a linear regression is a good approximation.

It is our impression that CLCT is more suitable for the presently investigated case of the moat flow, where small individual structures such as UV bright points and MMFs move through their surroundings, because smaller correlation areas can be selected. 
H. Balthasar and K. Muglach: The three-dimensional structure of sunspots. II.
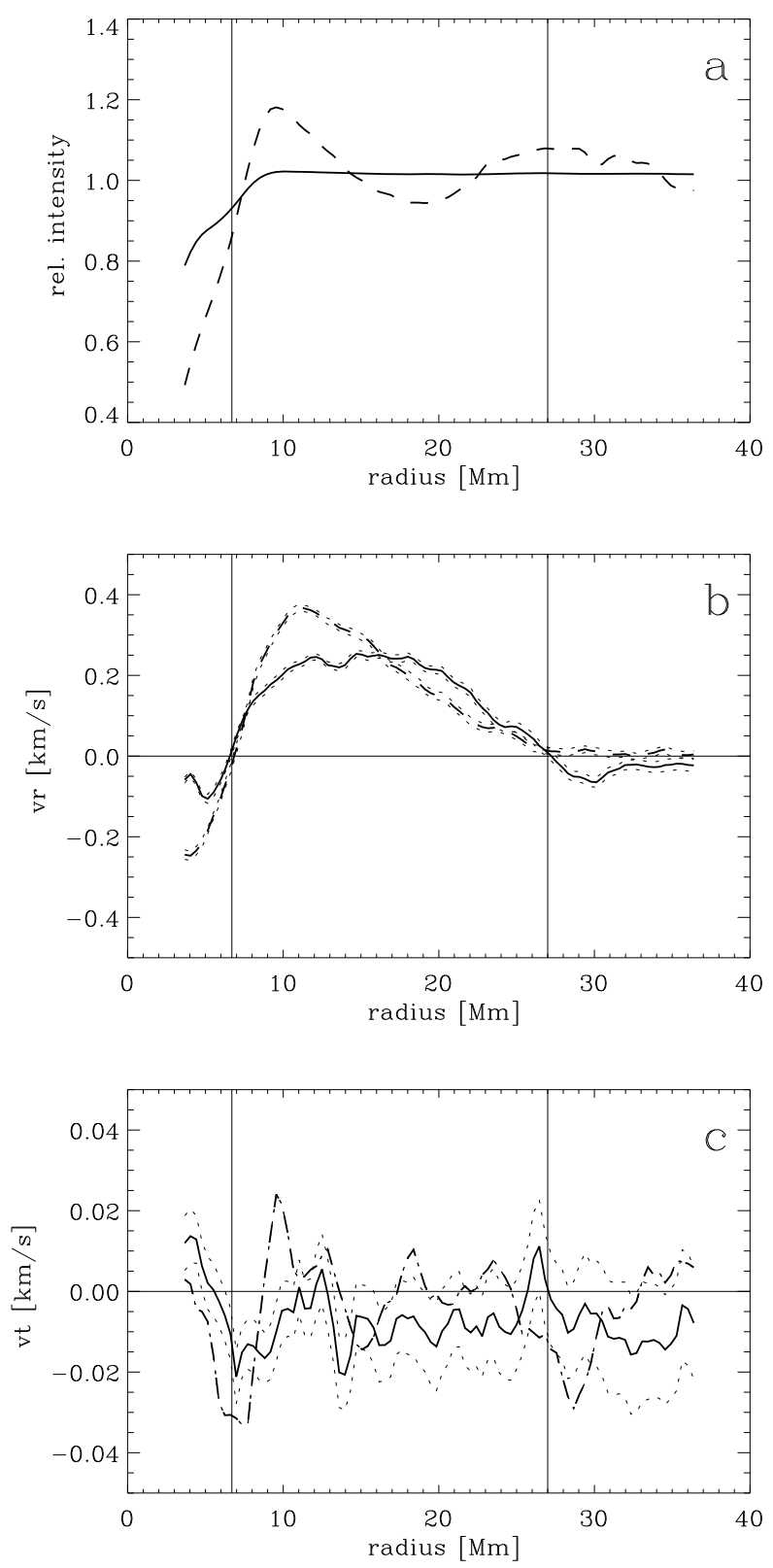

Fig. 3. Radial dependence of relative intensities a), radial velocity component b), and tangential velocity component $\mathbf{c}$ ), averaged over the azimuth angle $\varphi$. WL data are given by the solid lines, UV data by dashed ones. Error ranges are indicated by the dotted lines. Vertical lines mark the outer boundaries of penumbra and moat. The zero position of the radius corresponds to the center of the spot marked in Fig. 1. The box size used in this figure is 4 . Note the different scales for radial and tangential velocity components!

\section{Discussion}

In the inner moat, the proper motions measured in the UV are dominated by the bright points, which probably correspond to MMFs. MMFs exhibit high velocities of up to $2 \mathrm{~km} \mathrm{~s}^{-1}$ (see Hagenaar \& Shine 2005), which are higher than velocities obtained from the granular pattern $\left(0.3-0.5 \mathrm{~km} \mathrm{~s}^{-1}\right.$; see Sobotka \& Roudier 2007; and Vargas Domínguez et al. 2008) or from spectroscopy (Balthasar et al. 1996). The UV-bright points are much rarer in the outer moat. Thus, the faint granular pattern affects the LCT results more than in the inner moat. The extension of the moat of this spot is quite large, i.e., four times the radius of
Table 1. Linear regression fits ${ }^{a}$ for the radial velocities in the moat from FLCT versus those from CLCT.

\begin{tabular}{lrrcc}
\hline \hline$\lambda \lambda$ & $\begin{array}{r}\text { box } \\
\text { (CLCT) }\end{array}$ & $\begin{array}{r}\sigma \\
\text { (FLCT) }\end{array}$ & $a_{0}$ & $a_{1}$ \\
\hline WL & 4 & 4 & $0.036 \pm 0.003$ & $0.563 \pm 0.014$ \\
UV & 4 & 4 & $0.054 \pm 0.004$ & $0.781 \pm 0.018$ \\
WL & 10 & 10 & $0.041 \pm 0.008$ & $0.819 \pm 0.019$ \\
UV & 10 & 10 & $0.093 \pm 0.007$ & $0.678 \pm 0.018$ \\
WL & 15 & 15 & $0.030 \pm 0.008$ & $0.784 \pm 0.018$ \\
UV & 15 & 15 & $0.083 \pm 0.007$ & $0.685 \pm 0.019$ \\
WL & 12 & 4 & $0.013 \pm 0.003$ & $0.416 \pm 0.010$ \\
UV & 12 & 4 & $0.029 \pm 0.004$ & $0.558 \pm 0.012$ \\
\hline
\end{tabular}

Notes. ${ }^{(a)}$ Given are the coefficients from equation $v_{\mathrm{r}}(\mathrm{FLCT})=a_{0}+$ $a_{1} v_{\mathrm{r}}(\mathrm{CLCT})$.

the spot. Statistically, on average, only a factor of 1 or 2 is expected, as found by Brickhouse \& Lites (1988) and Sobotka \& Roudier (2007). Although this spot was in its decaying phase, its area remained almost constant for several days. Therefore, we cannot interprete the extension of the moat as a relict of an earlier larger spot. It is more likely to be related to the magnetic configuration, since the moat flow fills the space given by the magnetic network. Whether this extended moat affects the decay of the spot remains speculative.

Originally, MMFs were assumed to be pieces of the sunspot that have been detached from the main spot and are being swept away by the radial outflow of the convection around the spot (as in supergranules, e.g., Simon \& Leighton 1964). In their seminal paper, Harvey \& Harvey (1973) find that MMFs transport net flux away at the same rate as the flux decay of the spot. They, therefore, consider MMFs as a signature of sunspot decay (Kubo et al. 2007, 2008).

On the other hand, several studies have proposed that (at least some) MMFs are an extension of both the penumbra and the Evershed flow (Zhang et al. 2003; Sainz Dalda \& Martínez Pillet 2005; Cabrera Solana et al. 2006; Vargas Domínguez et al. 2007). According to this interpretation, MMFs remain magnetically connected to the central sunspot.

MMFs are identified with their magnetic signature. In Paper I, the magnetic properties of this active region were determined from full Stokes polarimetry. These data were taken on the same day, but after the TRACE sequences. Therefore, we cannot use them to identify MMFs directly by their polarization signature. In addition, we do not have high resolution MDI magnetograms in hand for the time of the TRACE observations. Nevertheless, we can use the bright points in UV as proxies of the MMFs (compare the averaged UV images and MDI magnetograms in Muglach 2003). Thus, the flow velocities in the UV represent the velocities of the magnetic structures in the moat. Magnetic field strengths derived from full Stokes polarimetry of MMFs are mostly well below $1000 \mathrm{G}$ (Kubo et al. 2007). The mechanism of flux expulsion can accumulate a field to the limit approximately given by the equipartition of the magnetic energy density of flux tubes and the kinetic energy density of the granular flows (Parker 1963). This equipartition field strength is around $\sim 500 \mathrm{G}$, similar to the field strengths of MMFs. Hence, the MMFs cannot significantly alter the granular flow field. We conclude that the flow velocities in WL represent the outward flow of the unmagnetized plasma, as the intensity contrast in WL is mainly caused by the granular pattern.

In Fig. 4b, we see that the radial velocities in WL and UV are different and this difference is larger than the error in the 
A\&A 511, A67 (2010)
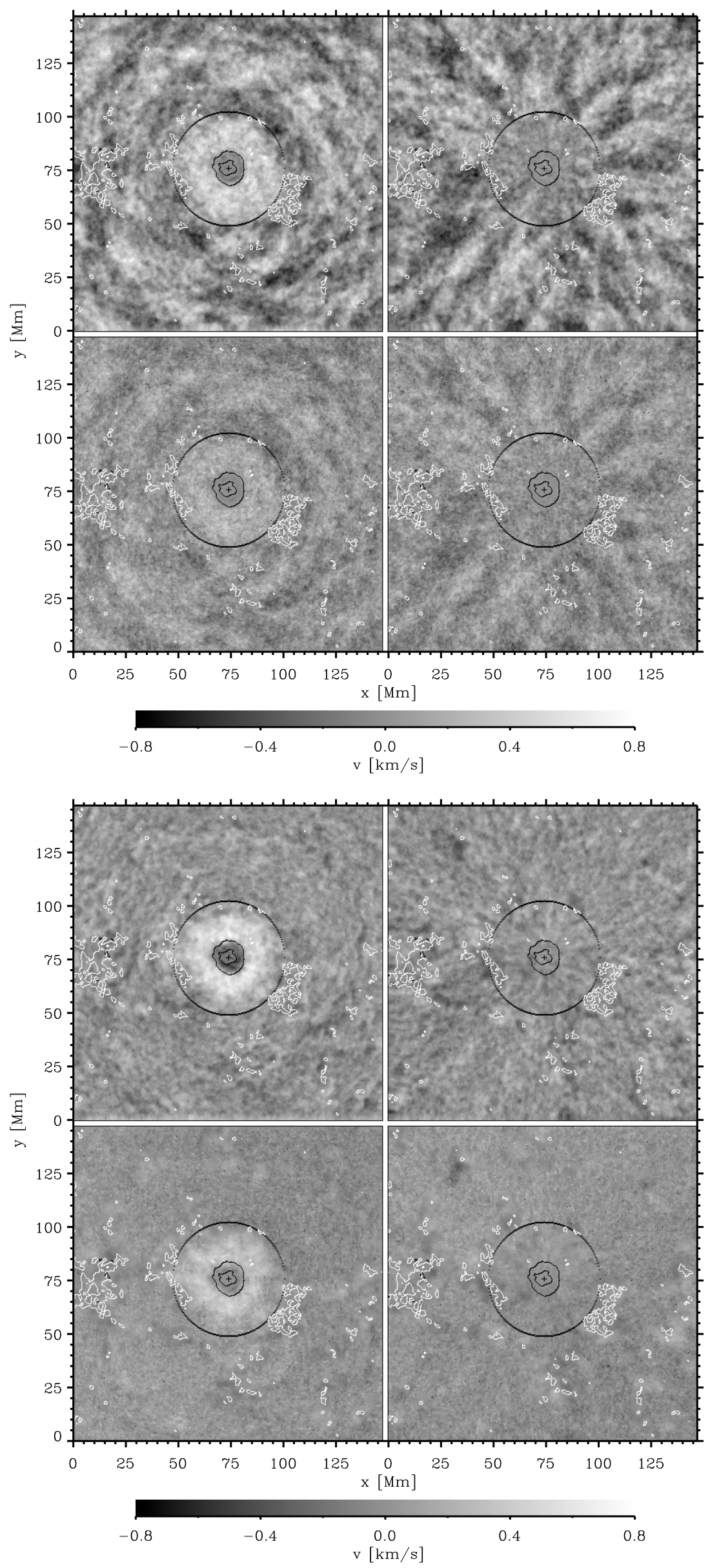

Fig. 4. Radial (left) and tangential (right) horizontal velocities for the WL series from CLCT (top) and from FLCT (bottom), obtained with a box size parameter of 12 pixels for CLCT and $\sigma=4$ pixels for FLCT.

Fig. 5. Same as Fig. 4 but for UV series. 
H. Balthasar and K. Muglach: The three-dimensional structure of sunspots. II.

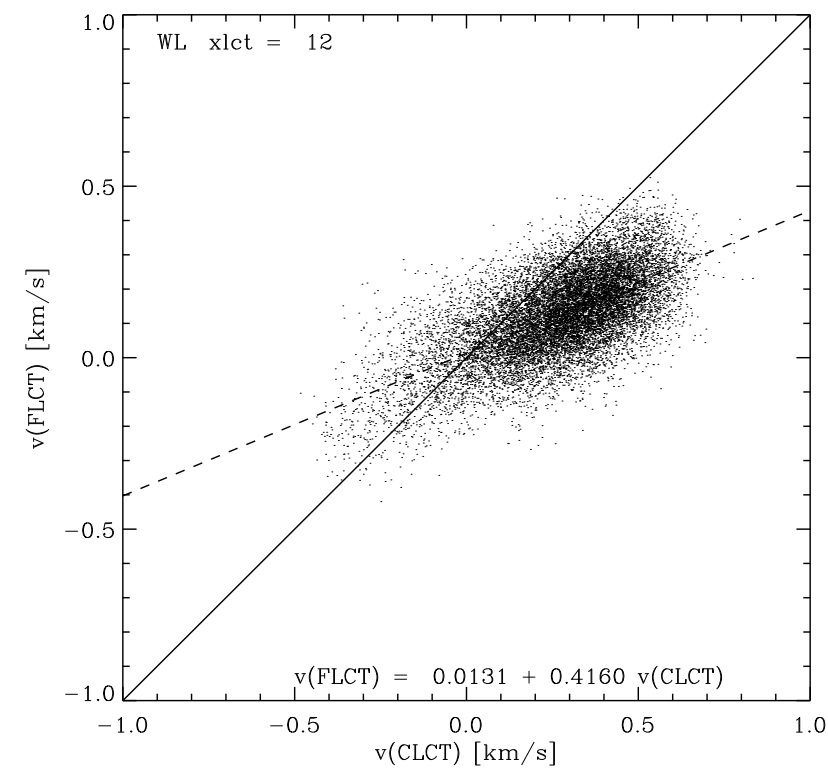

Fig. 6. Comparison of the derived radial velocities from FLCT versus CLCT, restricted to the moat area. The dashed line represents the linear regression fit given in the lower part of the figure. The solid line is the 1:1 diagonal. The box size (CLCT) is 12 pixels and the width of the Gaussian bell, $\sigma$, (FLCT) is four pixels.

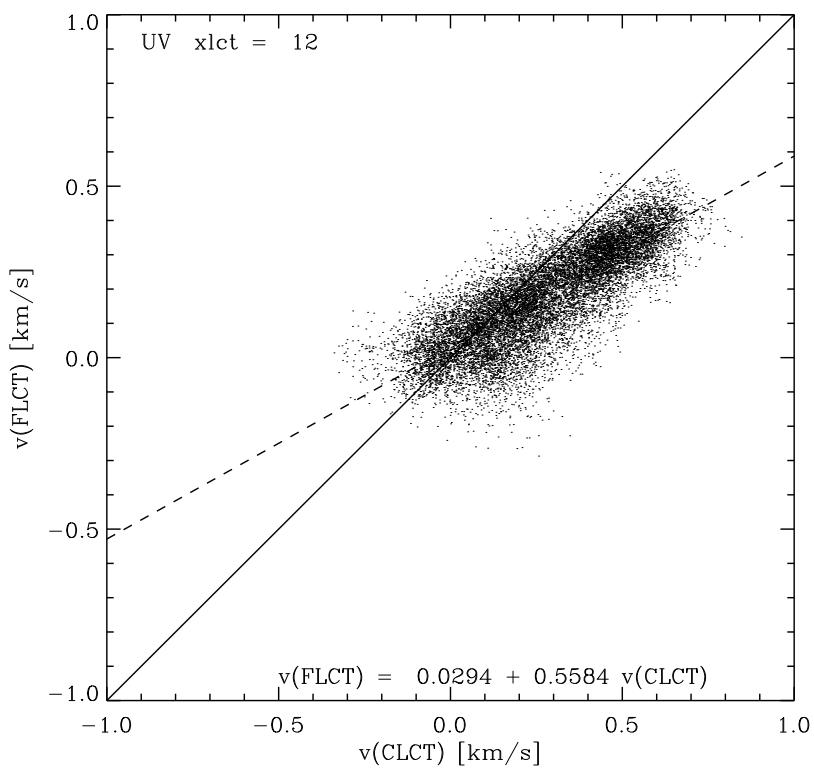

Fig. 7. Same as Fig. 6 but for UV.

measurement. In the UV, the apparent movement of the bright points dominate the LCT results. This suggests that the MMFs are not simply swept along the moat outflow in a completely passive way, but any detailed explanation of the physical processes causing the faster motion of the MMFs would be rather speculative at the present time.

\section{Conclusions}

Our most important results are summarized as follows:

1. The moat is filled with a horizontal outflow detectable in WL as well as in UV. In the inner moat, we detected higher velocities in UV than in WL and the converse for the outer moat. This outflow ends for both wavelength ranges at about
$27 \mathrm{Mm}$ (four times the radius of the spot) away from the center of the spot.

2. In the penumbra, we detected inward motions, mainly in the UV. However, the TRACE pixel size of 0.5 is too coarse to perform a sophisticated investigation of penumbral fine structures.

3. Outside the moat, the velocity pattern of the supergranulation was observed in WL as ring structures in the radial component and as alternate positive and negative azimuthal values in the tangential component.

4. CLCT was found to infer larger velocities than FLCT, and FLCT was found to probably have limitations to its applicability if features moving at different velocities occur close to each other.

Higher spatial resolution is needed in future investigations, and strictly simultaneous spectropolarimetric observations would help to clarify many open questions such as the relation between moat flow and Evershed effect, or to what extent the UV bright points are identical to MMFs. For the visible range, these observations will become possible with the next generation of groundbased telescopes (e.g., GREGOR, Volkmer et al. 2007), which are currently under construction.

Acknowledgements. We acknowledge the open data policy of the TRACE team. We also thank G. Fisher and B. Welsch for help with the FLCT code and A. Antunes for running it on the NRL parallel computer cluster.

\section{References}

Balthasar, H., \& Gömöry, P. 2008, A\&A, 488, 1085 (Paper I)

Balthasar, H., \& Schleicher, H. 2008, A\&A, 481, 811

Balthasar, H., Schleicher, H., Bendlin, C., \& Volkmer, R. 1996, A\&A, 315, 603

Bovelet, B., \& Wiehr, E. 2003, A\&A, 412, 249

Brickhouse, N. S., \& LaBonte, B. J. 1988, Sol. Phys., 115, 43

Cabrera Solana, D., Bellot Rubio, L. R., Beck, C., \& del Toro Iniesta, J. C. 2006, ApJ, 649, L41

Choudhary, D. P., \& Balasubramaniam, K. S. 2007, ApJ, 664, 1228

Deng, N., Xu, Y., Yang, G., et al. 2006, ApJ, 644, 1278

Deng, N., Choudhary, D. P., Tritschler, A., et al. 2007, ApJ, 671, 1013

Fisher, G. H., \& Welsch, B. T. 2008, in Subsurface and Atmospheric Influences on Solar Activity, ASP Conf. Ser., 383, 373

Georgakilas, A. A., Christopoulou, E. B., \& Koutchmy, S. 2000, A\&A, 363, 306 Giovanelli, R. G. 1972, Sol. Phys., 27, 71

Hagenaar, H. J., \& Shine, R. A. 2005, ApJ, 635, 659

Handy, B., Acton, L. W., Kankelborg, C. C., et al. 1999, Sol. Phys., 187, 229

Harvey, K. L., \& Harvey, J. 1973, Sol. Phys., 28, 61

Kubo, M., Shimizu, T., \& Tsuneta, S. 2007, ApJ, 659, 812

Kubo, M., Lites, B. W., Shimizu, T., \& Ichimoto, K. 2008, ApJ, 686, 1447

Lin, C.-H., Banerjee, D., O’Shea, E., \& Doyle, J. G. 2006, A\&A, 460, 597

Muglach, K. 2003, A\&A, 401, 685

November, L. J., \& Simon, G. W. 1988, ApJ, 333, 427

Parker, E. N. 1963, ApJ, 138, 552

Ravindra, B. 2006, Sol. Phys., 237, 297

Rimmele, T. R. 1997, ApJ, 490, 458

Sainz Dalda, A., \& Martínez Pillet, V. 2005, ApJ, 632, 1176

Sheeley, N. R. 1969, Sol. Phys., 9, 347

Sheeley, N. R. 1972, Sol. Phys., 25, 98

Simon, G. W., \& Leighton, R. B. 1964, ApJ, 140, 1120

Sobotka, M., \& Roudier, T. 2007, A\&A, 472, 277

Uitenbroek, H. 2004, in SOHO 13 Proceedings, ESA SP-547, 107

Vargas Domínguez, S., Bonet, J. A., Martínez Pillet, V., et al. 2007, ApJ, 660, L165

Vargas Domínguez, S., Rouppe van der Voort, L., Bonet, J. A., et al. 2008, ApJ, 679,900

Vernazza, J. E., Avrett, E. H., \& Loeser, R. 1981, ApJS, 45, 635

Volkmer, R., v. d. Lühe, O., Kneer, F. et al. 2007, in Modern Solar Facilities Advanced Solar Science, Universitätsverlag Göttingen, ed. F. Kneer, K. G. Puschmann, \& A. D. Wittmann, 39

Welsch, B. T., Abbett, W. P., DeRosa, M. L., et al. 2007, ApJ, 670, 1434

Zhang, J., Solanki, S. K., \& Wang, J. 2003, A\&A, 399, 755

Zhang, J., Solanki, S. K., Woch, J., \& Wang, J. 2007, A\&A, 471, 1035

Zuccarello, F., Romano, P., Guglielmino, S. L., et al. 2009, A\&A, 500, L5 